\title{
Histoplasmose pulmonar grave em um gato doméstico: tratamento com Anfotericina B e Itraconazol
}

\author{
Acute Pulmonary Histoplasmosis in a Domestic Cat Treated with Amphotericin B and Itraconazole \\ Mariana Palha de Brito Jardim', Monique Lourenço e Silva², Amanda Chaves de Jesus', Gabriela de Carvalho Cid ${ }^{3}$, \\ Cristiane Brandão Damico², Carla Regina Gomes Rodrigues dos Santos' ${ }^{1}$ \& Heloisa Justen Moreira de Souza ${ }^{4}$
}

\begin{abstract}
Background: Histoplasmosis is a systemic mycosis whose etiologic agent is the fungus Histoplasma capsulatum. This fungal infection, which is the second most frequent systemic mycotic fungal disease in felines in the United States, has rarely been found in cats in Brazil. This paper reports on a case of acute pulmonary histoplasmosis in a domestic cat treated with oral itraconazole associated with amphotericin B administered subcutaneously. This treatment resulted in clinical remission of the patient's symptoms, as evidenced by radiographic follow-ups.

Case: A domestic cat suffering from acute dyspnea was taken to a veterinary clinic. The animal was subjected to emergency oxygen therapy, and kept at rest through sedation with midazolam. A physical examination revealed normally colored mucosa, $8 \%$ dehydration, bristly fur, body condition score $2 / 9$, tachypnea with respiratory rate of 100 breaths per minute and expiratory dyspnea. The radiographic examination showed marked opacification of all the pulmonary fields, with a mixed pattern (interstitial and alveolar) of heterogeneous appearance and diffuse distribution, which are changes consistent with an inflammatory infectious process (pneumonia). A cytological analysis of the pleural fluid revealed round to oval-shaped intracytoplasmic structures, varying in size from 2 to $4 \mu \mathrm{m}$, inside foamy macrophages, consistent with Histoplasma capsulatum. Based on the diagnosis of pulmonary histoplasmosis, and in view of the patient's acute respiratory distress, it was decided to treat the cat using itraconazole associated with amphotericin B. Itraconazole was administered orally at a dose of $100 \mathrm{mg} /$ cat every $24 \mathrm{~h}$, while amphotericin B was administered subcutaneously at a dose of $0.5 \mathrm{mg} / \mathrm{kg}$, combined with $100 \mathrm{~mL}$ of sodium chloride $0.9 \%$ and $100 \mathrm{~mL}$ of $5 \%$ glycated serum, with monitoring of serum concentrations of symmetric dimethylarginine (SDMA). Amphotericin B was administered every $48 \mathrm{~h}$ up to the $7^{\text {th }}$ dose, followed by three times a week up to the $11^{\text {th }}$ dose, and then twice a week up to the $16^{\text {th }}$ dose. The animal was released from the veterinary hospital 10 days after its admission, showing a considerably improved respiratory condition and a respiratory rate of 40 breaths per minute. Continuation of the antifungal treatment with itraconazole was prescribed, maintaining the same dose and frequency of administration until a new recommendation would be made, as well as frequent return visits for the administration of amphotericin B and reassessment of the animal. The administration of amphotericin B was discontinued when the animal's respiratory condition stabilized; hence, the feline received a total of 16 doses of the drug. The animal showed remission of clinical respiratory symptoms, confirmed by follow-up chest X-rays, which revealed significantly less radiodense pulmonary parenchyma in response to treatment with itraconazole and amphotericin B. Five months after beginning its treatment, the cat is being treated orally with itraconazole $(100 \mathrm{mg} / \mathrm{cat}$, every $24 \mathrm{~h})$, without recrudescence of the clinical symptoms.

Discussion: To date, six cases of feline histoplasmosis have been reported in Brazil, only one of which had pulmonary involvement and was treated successfully. The use of oral itraconazole associated with subcutaneous amphotericin B has proved to be effective for the treatment of acute pulmonary histoplasmosis in domestic cats. Note that a rapid diagnosis of pulmonary disease is essential in order to immediately implement a combined drug therapy linked with monitoring to ensure the protocol is safe for the patient.
\end{abstract}

Keywords: feline, histoplasma, antifungal, SDMA.

Descritores: felinos, histoplasma, antifúngico, SDMA. erinária, Universidade Federal Rural do Rio de Janeiro (UFRRJ), Seropédica, RJ, Brazil. ${ }^{2}$ Universidade Federal Fluminense (UFF), Niterói, RJ. CORRESPONDENCE: M.P.B. Jardim [jardim.marii@gmail.com - Tel.: +55 (21) 99318-7447]. Setor de Clínica Médica dos Gatos Domésticos, Instituto de Veterinária, UFRRJ. Rodovia BR 465, Km 07, s/n. Zona Rural. CEP 238990-000 Seropédica, RJ, Brazil. 


\section{INTRODUÇÃO}

A histoplasmose é uma micose sistêmica ocasionada pelo Histoplasma capsulatum, fungo dimórfico distribuído mundialmente na natureza [3].

O itraconazol é o fármaco de escolha para o tratamento da histoplasmose $[1,10]$. Em casos potencialmente graves de manifestação da doença, a terapia deve ser associada à utilização da anfotericina B. No entanto, há implicações colaterais no uso desta droga, principalmente devido ao seu potencial nefrotóxico $[7,15,16]$.

A histoplasmose é a segunda doença micótica sistêmica mais frequente em felinos nos Estados Unidos, contudo é pouco descrita em gatos no Brasil, onde não há casos de felinos com comprometimento pulmonar, confirmadamente associados ao Histoplasma capsulatum. Havendo assim uma escassez de estudos, que podem por sua vez refletir o subdiagnóstico da doença e a carência de métodos eficazes e seguros de tratamento $[3,5,21,22]$.

O objetivo deste estudo é relatar um caso de histoplasmose pulmonar grave em um felino doméstico tratado com itraconazol oral associado à anfotericina B por via subcutânea, onde a terapêutica empregada resultou em remissão clínica da sintomatologia apresentada pelo paciente, comprovada por acompanhamentos radiográficos.

\section{CASO}

Um gato doméstico, fêmea, não esterilizada, sem raça definida, com aproximadamente dois anos de idade e peso correspondente a 2,3 $\mathrm{kg}$ foi levada para atendimento em uma clínica veterinária exclusiva para o atendimento de felinos, localizada no município do Rio de Janeiro - RJ, com queixa principal de dispneia grave. Mediante obtenção do histórico pregresso foi verificado que a paciente possuía estilo de vida livre, em uma região com alta densidade populacional de pombos e morcegos, localizada na zona oeste da cidade do Rio de Janeiro.

$\mathrm{O}$ animal recebeu atendimento emergencial imediato devido ao quadro de insuficiência respiratória. O felino foi mantido sob oxigenoterapia e em repouso no gatil, assim, foi sedado com midazolam (Dormire $\left.{ }^{\circledR}\right)^{1}$, na dose de $0,1 \mathrm{mg} / \mathrm{kg}$, por via intramuscular, a cada 8 horas, evitando-se ao máximo a manipulação, em razão da instabilidade e angustia respiratória. Este protocolo foi instituído nos três primeiros dias de internação.
Na inspeção notou-se mucosas normocoradas, desidratação de $8 \%$, pelagem eriçada, escore de condição corporal 2/9, taquipneia, com valor de frequência respiratória igual a 100 movimentos por minuto e dispneia expiratória. Na palpação não foram observadas manifestações significativas e nem mesmo foi notado linfonodomegalia. À ausculta cardiopulmonar mostrou-se adequada a espécie. Não foi observado tosse, espirro ou presença de secreção nasal. No exame ultrassonográfico abdominal não foi vista alteração digna de nota.

O teste ELISA (Teste SNAP FIV/FeLV Combo) ${ }^{2}$ para o anticorpo da imunodeficiência felina e antígeno da leucemia felina revelaram-se negativos.

No exame radiográfico foi visualizada acentuada opacificação de todos os campos pulmonares, com padrão misto (intersticial e alveolar), de aspecto heterogêneo e distribuição difusa, alterações estas compatíveis com processo inflamatório/infeccioso (pneumonia) [Figura 2A].

O felino foi submetido à toracocentese após estabilização do quadro clínico respiratório, que se deu 12 h após admissão do paciente em regime de internação. Para realização do procedimento o animal foi mantido em plano anestésico superficial com propofol (Propovan $\left.{ }^{\circledR}\right)^{1}$, na dose de $4 \mathrm{mg} / \mathrm{kg}$ por via intravenosa, a fim de realizar-se citologia do fluido pleural para elucidação diagnóstica. Utilizou-se scalp n ${ }^{\circ} 23 \mathrm{G}$ (Scalp BD Asepto 23G) ${ }^{3}$ e seringa de $5 \mathrm{~mL}$ (Seringa Descartável SR 05 ML Luer Lock Sem Agulha $)^{4}$ e assim fora acessado o $7^{\circ}$ espaço intercostal e aspirado $1,0 \mathrm{~mL}$ de líquido pleural. À microscopia foi observada a presença de estruturas leveduriformes intracelulares do Histoplasma capsulatum em macrófagos, como pode ser visto na Figura 1A e 1B.

A terapia medicamentosa instituída baseou-se na associação do itraconazol (Itralex $\left.100 \mathrm{mg}^{\circledR}\right)^{5} \mathrm{com}$ a anfotericina B (Anforicin B 50mg $\left.{ }^{\circledR}\right)^{1}$, em virtude do severo quadro clínico respiratório e grau de comprometimento pulmonar. O itraconazol foi utilizado na dose de $100 \mathrm{mg} /$ gato, por via oral, a cada $24 \mathrm{~h}$ e a anfotericina B na dose de $0,5 \mathrm{mg} / \mathrm{kg}$, por via subcutânea, a ser administrada juntamente a $100 \mathrm{~mL}$ de cloreto de sódio 0,9\% (Cloreto de Sódio 0,9\%) e $100 \mathrm{~mL}$ de glicose 5\% (Glicose 5\%), mediante acompanhamento do SDMA sérico (IDEXX SDMA $\left.{ }^{\mathrm{TM}}\right)^{2}$. A aplicação de anfotericina B ocorreu a cada $48 \mathrm{~h}$ até a $7^{\mathrm{a}}$ dose, três vezes por semana até a $11^{\mathrm{a}}$ dose e duas vezes por semana até a $16^{\mathrm{a}}$ dose. 
O felino recebeu alimentação úmida hipercalórica e tratamento de suporte para náusea e estimulação de apetite devido à hiporexia.

No quinto dia de terapia com itraconazol e anfotericina $\mathrm{B}$, foi aplicado no felino acetato de metilprednisolona (Depo-medrol $\left.40 \mathrm{mg} / \mathrm{mL}^{\circledR}\right)^{7}$, na dose de $1,0 \mathrm{mg} / \mathrm{kg}$ por via intramuscular para diminuição do processo inflamatório pulmonar.
No $10^{\circ}$ dia de tratamento para histoplasmose o animal apresentou considerável melhora no que tange o comprometimento pulmonar á radiografia torácica, conforme pode ser visualizado na Figura 2B.

O paciente foi liberado da internação após 10 dias de admissão, com considerável melhora do padrão respiratório e frequência respiratória igual a 40 movimentos por minuto, assim foi prescrito a continuação do

Tabela 1. Acompanhamento laboratorial do felino com histoplasmose pulmonar grave.

\begin{tabular}{|c|c|c|c|}
\hline $\begin{array}{c}\text { Tratamento com } \\
\text { anfotericina B em dias }\end{array}$ & $\begin{array}{l}\text { Aplicação de } \\
\text { anfotericina B }\end{array}$ & $\begin{array}{l}\text { Concentração de SDMA e } \\
\text { níveis de creatinina sérica }\end{array}$ & Exames Hematológicos \\
\hline Dia 1 & $1^{\mathrm{a}}$ dose & -- & -- \\
\hline Dia 2 & -- & -- & $\begin{array}{c}\text { Ht 28\%; Leucócitos } 6.700 \text { mL } \\
\text { Proteína Plasmática Total: } 6,8 \mathrm{~g} / \mathrm{dL} \\
\text { Alteração: Eosinófilos } 0 \mathrm{~mL} \\
\text { Linfócitos } 1.206 \mathrm{~mL}\end{array}$ \\
\hline Dia 3 & $2^{\mathrm{a}}$ dose & -- & -- \\
\hline Dia 5 & $3^{\mathrm{a}}$ dose & -- & -- \\
\hline Dia 7 & $4^{\mathrm{a}}$ dose & -- & -- \\
\hline Dia 9 & $5^{\mathrm{a}}$ dose & $\begin{array}{c}\text { SDMA } 12 \mu \mathrm{g} / \mathrm{dL} \\
\text { Creatinina } 0,8 \mathrm{mg} / \mathrm{dL}\end{array}$ & $\begin{array}{c}\text { Ht 20\% (Anemia Normocítica } \\
\text { Normocrômica); } \\
\text { Leucócitos } 10.200 \mathrm{~mL} \\
\text { Proteína Plasmática Total: } 8,0 \mathrm{~g} / \mathrm{dL} \\
\text { Alteração: Eosinófilos } 0 \mathrm{~mL}\end{array}$ \\
\hline Dia 11 & $6^{\mathrm{a}}$ dose & -- & -- \\
\hline Dia 13 & $7^{\mathrm{a}}$ dose & -- & -- \\
\hline Dia 16 & $8^{\mathrm{a}}$ dose & -- & -- \\
\hline Dia 18 & $9^{a}$ dose & -- & -- \\
\hline Dia 20 & $10^{\mathrm{a}}$ dose & -- & -- \\
\hline Dia 23 & $11^{\mathrm{a}}$ dose & -- & -- \\
\hline Dia 25 & -- & $\begin{array}{c}\text { SDMA } 11 \mu \mathrm{g} / \mathrm{dL} \\
\text { Creatinina } 1,0 \mathrm{mg} / \mathrm{dL}\end{array}$ & $\begin{array}{l}\text { Ht 32\%; Leucócitos } 6.700 \mathrm{~mL} \\
\text { Proteína Plasmática Total: 8,0 g/dL } \\
\text { Alteração: Linfócitos } 1.474 \mathrm{~mL}\end{array}$ \\
\hline Dia 27 & $12^{\mathrm{a}}$ dose & -- & -- \\
\hline Dia 30 & $13^{\mathrm{a}}$ dose & -- & -- \\
\hline Dia 34 & $14^{\mathrm{a}}$ dose & -- & -- \\
\hline Dia 37 & $15^{\mathrm{a}}$ dose & -- & -- \\
\hline Dia 41 & $16^{\mathrm{a}}$ dose & $\begin{array}{c}\text { SDMA } 13 \mu \mathrm{g} / \mathrm{dL} \\
\text { Creatinina } 1,4 \mathrm{mg} / \mathrm{dL}\end{array}$ & -- \\
\hline Dia 83 & $\begin{array}{l}42 \text { dias pós } \\
\text { o término do } \\
\text { tratamento com } \\
\text { anfotericina } B\end{array}$ & $\begin{array}{c}\text { SDMA } 10 \mu \mathrm{g} / \mathrm{dL} \\
\text { Creatinina } 1,4 \mathrm{mg} / \mathrm{dL}\end{array}$ & $\begin{array}{l}\text { Ht 34\%; Leucócitos } 8.700 \text { mL } \\
\text { Proteína Plasmática Total: } 6,7 \mathrm{~g} / \mathrm{dL} \\
\text { Alteração: Eosinófilos } 87 \mathrm{~mL}\end{array}$ \\
\hline
\end{tabular}


tratamento antifúngico com itraconazol, mantendo-se a mesma dosagem e frequência de administração até novas recomendações, retornos frequentes a clínica para aplicação de anfotericina B conforme esquema descrito na Tabela 1 e reavaliação do animal. Nas revisões do paciente foram realizados exames radiográficos, hematológicos, bioquímicas séricas e dosagem de SDMA.

Ao $25^{\circ}$ dia de terapia antifúngica um novo exame radiográfico permitiu a constatação de significativa resposta do paciente ao tratamento instituído como visto na Figura 2C. O felino apresentou aumento de $0,250 \mathrm{~kg}$ de peso após 60 dias do início do protocolo medicamentoso.

As aplicações de anfotericina B foram interrompidas quando o quadro respiratório do animal se tornou estável, assim o gato recebeu no total, 16 doses da droga.

A tutora não observou e/ou relatou durante as avaliações do animal a ocorrência reações adversas às drogas utilizadas. As radiografias obtidas com 60, 83 e 165 dias de tratamento com itraconazol e anfotericina B podem ser visualizadas nas Figuras 2D, 2E e $2 \mathrm{~F}$ respectivamente. Transcorridos cinco meses após o início da terapia, o felino encontra-se em tratamento com itraconazol (100 mg/gato por via oral, a cada 24 h), sem recrudescência dos sinais clínicos.

\section{DISCUSSÃO}

Na radiografia torácica de animais com histoplasmose costuma-se visualizar alteração no parênquima pulmonar, principalmente o padrão intersticial, muito embora o comprometimento alveolar também possa ser identificado e corroborado a um prognóstico grave. A dispneia e a presença de ruídos pulmonares constituem as manifestações respiratórias mais frequentemente observadas e são associadas a um mau prognóstico da doença $[1,4,17]$.

O diagnóstico definitivo da histoplasmose dá-se através da citologia e/ou histopatologia, no entanto, tais formas diagnósticas são consideradas por vezes de risco e invasivas. Já a realização da cultura fúngica implica principalmente em risco biológico para os técnicos laboratoriais, além de ser pouco sensível. Outros métodos como a detecção antigênica de Histoplasma no soro, na urina e até mesmo em regiões corporais específicas como os olhos, requerem mais estudos para que possam ser de fato utilizados [1,6,9,11,16,23] assim como a Reação em Cadeia da Polimerase, empregada atualmente em alguns estudos [2,13].

O citodiagnóstico da histoplasmose pulmonar pode ser realizado através do lavado broncoalveolar, da colheita de líquido pleural ou da punção aspirativa por agulha fina a nível pulmonar [16]. No entanto, o comprometimento respiratório severo impossibilita a realização do lavado, assim busca-se através deste relato encorajar os clínicos a quanto à realização da toracocentense e colheita do líquido pleural nestas ocasiões, visando à obtenção do material de uma forma mais segura para o paciente.

A diferenciação citológica de outros patógenos fúngicos é facilitada pela morfologia e organização celular dos organismos Histoplasma capsulatum, visto que possuem pequeno tamanho e estão normalmente agrupados dentro de células [12].

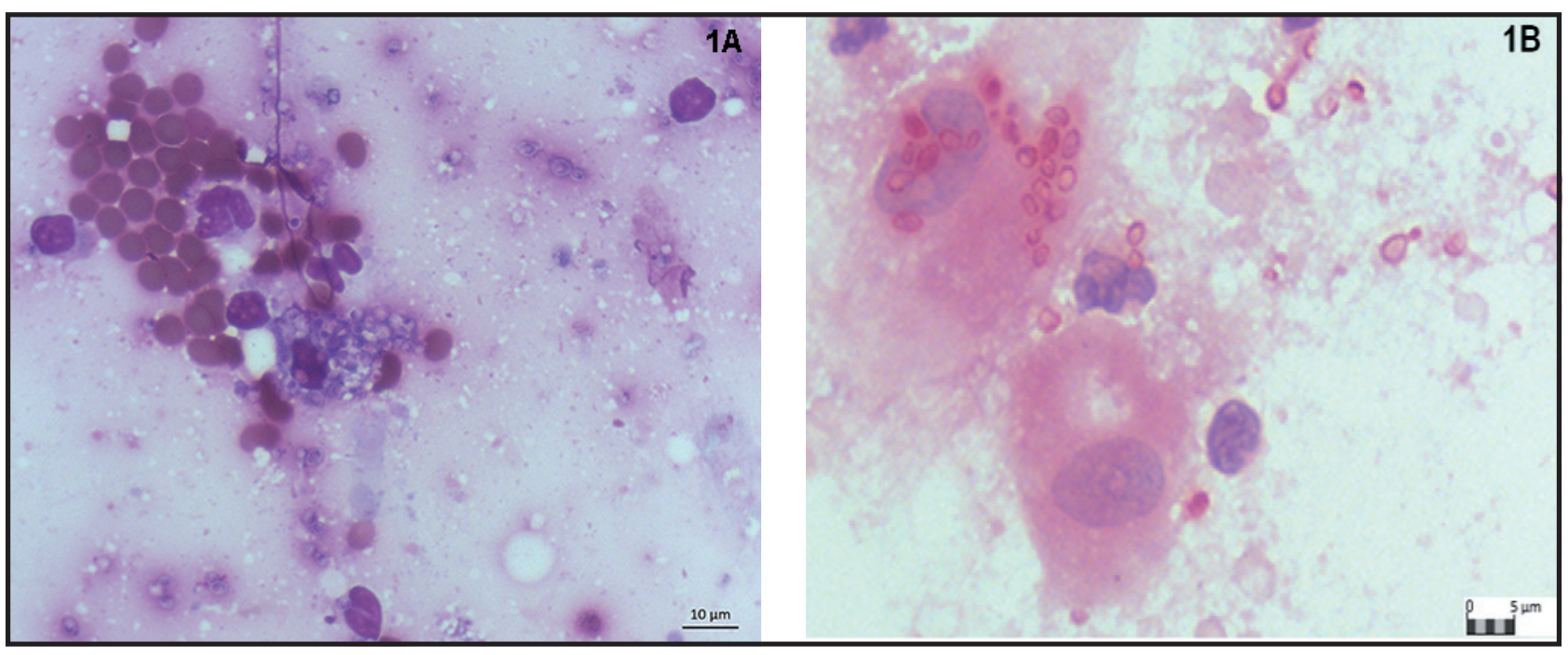

Figura 1. Exame citológico do líquido pleural de um felino com disfunção respiratória grave. 1A- Estruturas intracitoplasmáticas do agente Histoplasma capsulatum, de formato arredondado a ovalado medindo de 2 a $4 \mu \mathrm{m}$, em macrófago. [Coloração Giemsa - Obj. 100x]. 1B- Método histoquímico com marcação positiva para fungo. [Coloração ácido periódico de Schiff (PAS) - Obj. 200x]. 
M.P.B. Jardim, M.L. Silva, A.C. Jesus, et al. 2018. Histoplasmose pulmonar grave em um gato doméstico: tratamento com Anfotericina B e Itraconazol. Acta Scientiae Veterinariae. 46(Suppl 1): 334.

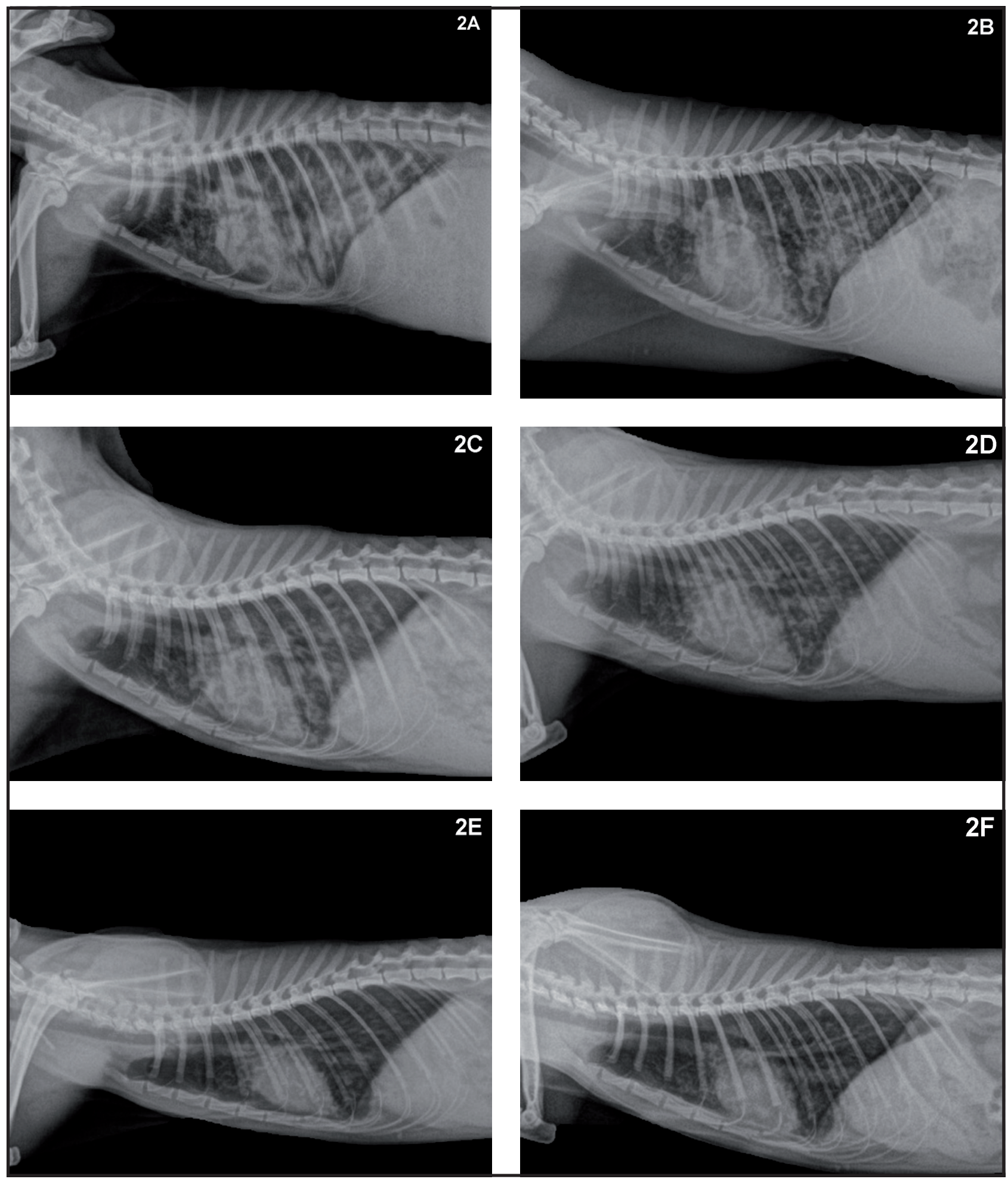

Figura 2. Radiografias torácicas laterais de um felino com histoplasmose pulmonar grave tratado com itraconazol associado à anfotericina B. 2A- Opacificação acentuada em todos campos pulmonares, de padrão misto (intersticial e alveolar), de aspecto heterogêneo e distribuição difusa, compatível com processo inflamatório/infeccioso (pneumonia), sinal de silhueta positivo - Radiografia realizada no primeiro dia de tratamento antifúngico. 2B- Radiopacidade moderada em parênquima pulmonar (tomando-se como base a imagem $2 \mathrm{~A}$ ) - Radiografia realizada no $10^{\circ}$ dia de tratamento. $2 \mathrm{C}$ - Marcação menos intensa dos lobos pulmonares (tomando-se como base a imagem 2B) - Radiografia realizada no $25^{\circ}$ dia de tratamento. 2D- Menor radiopacidade das campos pulmonares (tomando-se como base a imagem 2C) - Radiografia realizada no $60^{\circ}$ dia de tratamento. $2 \mathrm{E}$ - Opacificação discreta das áreas pulmonares, com clara visualização da silhueta cardíaca (tomando-se como base a imagem 2D) - Radiografia realizada no $83^{\circ}$ dia de tratamento. $2 \mathrm{~F}$ Radioluscência em arquitetura pulmonar com ligeira marcação, compatível com potencial remissão do quadro de penumonia fúngica, atrelada a resposta ao tratamento preconizado (tomando-se como base a imagem $2 \mathrm{~A}, 2 \mathrm{~B}, 2 \mathrm{C}, 2 \mathrm{D}$ e $2 \mathrm{E}$ ) - Radiografia realizada no $165^{\circ}$ dia de tratamento. 
O tratamento para felinos com histoplasmose baseia-se principalmente no uso do itraconazol na dose de $10 \mathrm{mg} / \mathrm{kg}$, a ser administrado por via oral, a cada 24 h. A terapia com o itraconazol é de longo prazo e perdura por aproximadamente seis meses. O itraconazol mostra-se mais efetivo que o fluconazol e o cetoconazol para o combate a afecção. Em casos potencialmente graves o itraconazol deve ser associado à anfotericina $\mathrm{B}$ na dose de $0,25 \mathrm{mg} / \mathrm{kg}$, a cada $48 \mathrm{~h}$, por via intravenosa, não devendo-se exceder a dose total de 4 a $16 \mathrm{mg} / \mathrm{kg}$. A anfotericina B pode ainda ser utilizada na dose de $0,5 \mathrm{mg} / \mathrm{kg}$, por via subcutânea, juntamente a solução de cloreto de sódio $0,9 \%$ e glicose. No entanto, é necessário considerar o potencial nefrotóxico deste agente antifúngico, mediante o cauteloso monitoramento da creatinina sérica [12,14,16,19,24].

A creatinina sérica somente se eleva após $75 \%$ da massa renal funcional ser perdida [18]. Desta forma caso a anfotericina B ocasionasse nefrotoxicidade, tal injúria somente seria verificada após um considerável comprometimento do parênquima renal, o que poderia afetar potencialmente a expectativa de vida do paciente.

O SDMA é uma molécula estável, originada a partir de proteínas intracelulares que desempenham um papel fundamental no metabolismo celular básico. Tal biomarcador é excretado principalmente pelos rins, correlacionando-se inversamente a taxa de filtração glomerular. A concentração sérica de dimetilarginina simétrica não sofre influência da massa muscular, ao contrário da creatinina sanguínea. Assim, possibilita de forma sensível e específica o diagnóstico prévio da doença renal, em comparação a rotineira mensuração de creatinina sérica. O SDMA foi incluído recentemente, no ano de 2015, dentre as diretrizes da Sociedade Internacional de Interesse Renal (IRIS), aplicadas a doença renal crônica em felinos $[8,18]$. O intervalo de referência de SDMA sérico para felinos estabelece limites de até $14 \mu \mathrm{g} / \mathrm{dL}$ [18].

Desta forma, o acompanhamento sérico de SDMA associado à utilização de anfotericina B constitui uma nova ferramenta disponível, para acompanhar com mais acurácia animais submetidos à terapia com tal droga, como fora relatado atualmente para o tratamento da esporotricose felina [20].

O uso de corticosteróides é considerado controverso, uma vez que estes medicamentos podem induzir a disseminação e o agravamento da infecção fúngica por suprimir a imunidade mediada por células, porém estas drogas também possuem grande potencial anti-inflamatório e auxiliam no tratamento da inflamação massiva induzida pela morte dos microrganismos fúngicos nas primeiras semanas de terapia. Portanto o emprego de corticosteroides só deve ser feito simultaneamente a administração dos antifúngicos ou em caso de piora das manifestações respiratórias após terapia antifúngica [12].

Até o momento foram relatados seis casos de histoplasmose felina a nível nacional, dentre os quais em apenas um houve envolvimento pulmonar e sucesso na terapia empregada $[3,5,21,22]$.

Devido a carência de relatos sobre a histoplasmose felina em âmbito nacional e conseguinte ausência de protocolos terapêuticos eficazes e seguros faz-se necessário à publicação de estudos que contenham informações substanciais sobre a doença, bem como quanto à forma mais adequada de tratamento a depender das manifestações associadas.

O uso do itraconazol oral associado à anfotericina B por via subcutânea mostrou-se eficaz para o tratamento da histoplasmose pulmonar grave no felino doméstico. Destaca-se a necessidade do rápido diagnóstico da doença pulmonar a fim da imediata instituição de uma terapêutica farmacológica combinada e atrelada a um monitoramento que torne o protocolo seguro para o paciente.

\section{MANUFACTURERS}

\footnotetext{
${ }^{1}$ Cristália Produtos Químicos e Farmacêuticos Ltda. Itapira, SP, Brazil. ${ }^{2}$ IDEXX Brasil Laboratórios Ltda. Sao Paulo, SP, Brazil.

${ }^{3}$ Becton Dickinson Indústrias Cirúrgicas Ltda. Juiz de Fora, MG, Brazil. ${ }^{4}$ Saldanha Rodrigues Ltda. Manaus, AM, Brazil.

${ }^{5}$ EMS Indústria Farmacêutica Ltda. São Bernardo do Campo, SP, Brazil.

${ }^{6}$ Fresenius Kabi Brasil Ltda. Aquiraz, CE, Brazil.

${ }^{7}$ Laboratórios Pfizer Ltda. Itapevi, SP, Brazil.
}

Declaration of interest. The authors report no conflicts of interest. The authors alone are responsible for the content and writing of the paper. 


\section{REFERENCES}

1 Aulakh A.K., Aulakh K.S. \& Troy G.C. 2012. Feline Histoplasmosis: A Retrospective Study of 22 Cases (1986-2009). Journal of the American Animal Hospital Association. 48(3): 182-187

2 Bernhardt A., Bomhard W.V., Antweiler E. \& Tintelnot K. 2015. Molecular identification of fungal pathogens in nodular skin lesions of cats. Medical Mycology. 53(2): 132-144.

3 Brilhante R.S.N., Coelho C.G.V., Sidrim J.J.C., Lima A.C., Ribeiro J.F., Cordeiro R.A., Castelo-Branco D.S.C.M., Gomes J.M.F., Simões-Mattos L., Mattos M.R.F., Beserra H.E.O., Nogueira G.C., Pinheiro A.Q. \& Rocha M.F.G. 2012. Feline Histoplasmosis in Brazil: Clinical and Laboratory Aspects and a Comparative Approach of Published Reports. Mycopathologia. 172(2-3): 193-197.

4 Brömel C. \& Sykes J.E. 2005. Histoplasmosis in Dogs and Cats. Clinical Techniques in Small Animal Practice. 20(4): 227-232.

5 Carneiro R.A., Lavalle G.E. \& Araújo R.B. 2005. Histoplasmose cutânea em gato: relato de caso. Arquivo Brasileiro de Medicina Veterinária e Zootecnia. 57(2): 158-161.

6 Cook A.K., Cunningham L.Y., Cowell A.K. \& Wheat L.J. 2012. Clinical evaluation of urine Histoplasma capsulatum antigen measurement in cats with suspected disseminated histoplasmosis. Journal of Feline Medicine and Surgery. 14(8): 512-515.

7 Gallis H.A., Drew R.H. \& Pickard W.W. 1990. Amphotericin B: 30 years of clinical experience. Reviews of infectious diseases. 12(2): 308-329.

8 Hall J.A., Yerramilli M., Obare E., LI J., Yerramilli M. \& Jewell D.E. 2017. Serum concentrations of symmetric dimethylarginine and creatinine in cats with kidney stones. Plos One. 12(4): 1-11.

9 Hanzlicek A.S., Meinkoth J.H., Renschler J.S., Goad C. \& Wheat L.J. 2016. Antigen Concentrations as an Indicator of Clinical Remission and Disease Relapse in Cats with Histoplasmosis. Journal of Veterinary Internal Medicine. 30(4): 1065-1073.

10 Hodges R.D., Legendre A.M., Adams L.G., Willard M.D., Pitts R.P., Monce K., Needels C.C. \& Ward H. 1994. Itraconazole for the Treatment of Histoplasmosis in Cats. Journal of Veterinary Internal Medicine. 8(6): 409-413.

11 Jarchow A. \& Hanzlicek A. 2015. Antigenemia without antigenuria in a cat with histoplasmosis. Journal of Feline Medicine and Surgery Open Reports. 1(2): 1-4.

12 Kerl M.E. 2003. Update on canine and feline fungal diseases. The Veterinary Clinics of North America. Small Animal Practice. 33(4):721-747.

13 Klang A., Loncaric I., Spergser J., Eigelsreiter S. \& Weissenböck H. 2013. Disseminated histoplasmosis in a domestic cat imported from the USA to Austria. Medicinal Mycology Case Reports. 23(2): 108-112.

14 Larsuprom L., Duangkaew L., Kasorndorkbua C., Chen C., Chindamporn A. \& Worasilchai N. 2017. Feline cutaneous histoplasmosis: The first case report from Thailand. Medical Micology Case Reports.18: 28-30.

15 Li R.K., Ciblak M.A., Nordoff N., Pasarell L., Warnock D.W. \& Mcginnis M.R. 2000. In Vitro Activities of Voriconazole, Itraconazole, and Amphotericin B against Blastomyces dermatitidis, Coccidioides immitis, and Histoplasma capsulatum. Antimicrobial Agents and Chemoterapy. 44(6): 1734-1736.

16 Lloret A., Hartmann K., Pennisi M.G., Ferrer L., Addie D., Belák S., Boucrat-Baralon C., Egberink H., Frymus T., Gruffydd-Jones T., Hosie M.J., Lutz H., Marsilio F., M Östl K., Radford A.D., Thiry E., Truyen U. \& Horzinek M.C. 2013. Rare systemic mycoses in cats: blastomycosis, histoplasmosis and coccidioidomycosis: ABCD guidelines on prevention and management. Journal of Feline Medicine and Surgery. 15(7): 624-627.

17 Ludwig H.C., Hanzlicek A.S., Kukanich K.S. \& Payton M.E. 2017. Candidate prognostic indicators in cats with histoplasmosis treated with antifungal therapy. Journal of Feline Medicine and Surgery. [01 Dec 2017:1098612X17746523].

18 Relford R., Robertson J. \& Clements C. 2016. Symmetric Dimethylarginine Improving the Diagnosis and Staging of Chronic Kidney Disease in Small Animals. The Veterinary Clinics of North America. Small Animal Practice. 46(6): 941-960.

19 Renschler J.S., Norsworthy G.D., Rakian R.A., Rakian A.I., Wheat L.J. \& Hanzlicek A.S. 2017. Reduced susceptibility to fluconazole in a cat with histoplasmosis. Journal of Feline Medicine and Surgery Open Reports. 28(2): 1-4.

20 Santos C.R.G.R., Souza H.J.M., Farias L.F., Jardim M.P.B., Carrasco L.P.S. \& Moreira C.M. 2018. Uso de Anfotericina B por via intralesional associada ao itraconazol oral para tratamento de recidiva de esporotricose nasal em um gato - relato de caso. Revista Clínica Veterinária. 132(1): 48-52. 
21 Silveira E., Mottin I.B., Ehlers A. \& Marques S.M.T. 2016. Histoplasmose felina disseminada: relato de um caso. PUBVET. 10(3): 207-211.

22 Souza E.W., Gremião I.D.F., Pereira S.A., Cunha S.C.S. \& Borba C.M. 2015. Feline histoplasmosis: a case of supraorbital involvement. Revista Brasileira de Ciência Veterinária. 22(2): 77-80.

23 Smith K.M., Strom A.R., Gilmour M.A., Ladouceur E., Reilly C.A., Byrne B.A., Affolter V.K., Sykes J.E. \& Maggs D.J. 2017. Utility of antigen testing for the diagnosis of ocular histoplasmosis in four cats: a case series and literature review. Journal of Feline Medicine and Surgery. 19(10): 1110-1118.

24 Taylor A.R., Barr J.W., Hokamp J.A., Johnson M.C. \& Young B.D. 2012. Cytologic Diagnosis of Disseminated Histoplasmosis in the Wall of the Urinary Bladder of a Cat. Journal of the American Animal Hospital Association. 48(3): 203-208. 